\title{
Les NTIC et la formation à distance : vers une industrialisation insolite?
}

\section{ICTs and Distance Education: Groundbreakers in Industrialization? \\ Las NTIC y la formación a distancia : ¿hacia una industrialización insólita?}

\section{Patrick Guillemet et Guy Provost}

Volume 27, numéro 2, automne 1999

Les technologies de l'information et de la communication et leur avenir en éducation

URI : https://id.erudit.org/iderudit/1080492ar

DOI : https://doi.org/10.7202/1080492ar

Aller au sommaire du numéro

Éditeur(s)

Association canadienne d'éducation de langue française

ISSN

0849-1089 (imprimé)

1916-8659 (numérique)

Découvrir la revue

Citer cet article

Guillemet, P. \& Provost, G. (1999). Les NTIC et la formation à distance : vers une industrialisation insolite? Éducation et francophonie, 27(2), 39-60.

https://doi.org/10.7202/1080492ar
Résumé de l'article

Inspiré par les études traitant de l'industrialisation de la formation, qui analysent le rôle joué par les nouvelles technologies de l'information et de la communication (NTIC), ce texte examine dans quelle mesure les questions soulevées par ce courant de recherche trouvent une résonance dans la pratique de la formation à distance. Il aborde notamment la question des gains de productivité attendus de leur introduction, la confrontation entre la logique marchande et la logique civique qui l'accompagne et la nature de la médiation pédagogique dans les nouveaux environnements d'apprentissage. Par ailleurs, sont mis en évidence d'autres paramètres qui conditionnent le succès de l'implantation des NTIC, par exemple la possibilité de formaliser les contenus, les conditions de travail qui accompagnent l'insertion des technologies et les compromis à effectuer entre les exigences pédagogiques et les contraintes techniques et financières. Prenant acte de la pénétration de certaines technologies, ce texte laisse ainsi entrevoir l'émergence d'une industrialisation insolite prenant la forme d'un " artisanat industriel " peut-être porteur de gains pédagogiques appréciables.

Tous droits réservés ( $\odot$ Association canadienne d'éducation de langue française, Ce document est protégé par la loi sur le droit d'auteur. L’utilisation des 1999 services d’Érudit (y compris la reproduction) est assujettie à sa politique d'utilisation que vous pouvez consulter en ligne.

https://apropos.erudit.org/fr/usagers/politique-dutilisation/ 


\title{
Les NTIC et la formation à distance: vers une industrialisation insolite?
}

\author{
Patrick GUILLEMET \\ Télé-université, Québec, Canada \\ Guy PROVOST \\ Télé-université, Québec, Canada
}

\section{RÉSUMÉ}

Inspiré par les études traitant de l'industrialisation de la formation, qui analysent le rôle joué par les nouvelles technologies de l'information et de la communication (NTIC), ce texte examine dans quelle mesure les questions soulevées par ce courant de recherche trouvent une résonance dans la pratique de la formation à distance. Il aborde notamment la question des gains de productivité attendus de leur introduction, la confrontation entre la logique marchande et la logique civique qui l'accompagne et la nature de la médiation pédagogique dans les nouveaux environnements d'apprentissage. Par ailleurs, sont mis en évidence d'autres paramètres qui conditionnent le succès de l'implantation des NTIC, par exemple la possibilité de formaliser les contenus, les conditions de travail qui accompagnent l'insertion des technologies et les compromis à effectuer entre les exigences pédagogiques et les contraintes techniques et financières. Prenant acte de la pénétration de certaines technologies, ce texte laisse ainsi entrevoir l'émergence d'une industrialisation insolite prenant la forme d'un «artisanat industriel» peut-être porteur de gains pédagogiques appréciables. 
ABSTRACT

ICTs and Distance Education: Groundbreakers in Industrialization?

Patrick GUILLEMET et Guy PROVOST

Télé-université, Quebec City, Canada

This article takes as its starting point studies on the role played by information and communications technologies (ICTs) in the industrialization of education. Its objective is to determine to what the extent the issues raised by this research are also present in distance education. It deals notably with the productivity increases ICTs have been expected to bring about, as well as the confrontation caused by new learning environments between the market (and public) mentality and teaching. The article also describes other parameters necessary for the success of ICTs, for example, the formalization of content, the work conditions associated with these technologies, and the compromises to be made between pedagogical requirements and technical and financial constraints. Taking as examples the implementation of specific technologies, the article demonstrates the emergence of a wholly new kind of industrialization, indeed, a kind of "cottage» industrialization that may lead to significant advances in education.

\section{RESUMEN}

\section{Las NTIC y la formación a distancia : ¿hacia una industrialización insólita?}

Patrick GUILLEMET et Guy PROVOST

Télé-université, Quebec, canada

Inspirándose en los estudios sobre la industrialización de la formación y aquellos que analizan el rol de las nuevas tecnologías de la información y la comunicación (NTIC), este articulo examina en que medida las cuestiones que hace surgir esta corriente investigativa repercuten en el ejercicio de la formación a distancia. Especialmente se aborda la cuestión del aumento de la productividad que su introducción permite esperar, la confrontación entre la lógica mercantil y la lógica cívica que lo acompaña y la naturaleza de la mediación pedagógica en los nuevos entornos de aprendizaje. Por otra parte se ponen en evidencia otros parámetros que condicionan el éxito en la implantación de las NTIC, como por ejemplo, la posibilidad de formalizar los contenidos, las condiciones de trabajo que surgen con la inserción de las tecnologías y los compromisos que se tienen que establecer entre las exigencias pedagógicas y las presiones técnicas y financieras. Constando la penetración de ciertas tecnologías, el articulo permite entrever el surgimiento de una industrialización insólita que toma la forma de un «artesanado industrial» probablemente portador de beneficios pedagógicos apreciables. 


\section{Des NTIC résolument francophones}

S'intéresser aux NTIC dans le cadre d'une revue orientée vers l'éducation et la francophonie, c'est constater d'emblée que toutes les activités humaines et sociales sont modelées et transformées par ces instruments d'organisation, de traitement et de diffusion des connaissances. Et, bien sûr, l'éducation, vecteur privilégié de la transformation sociale, doit répondre à des attentes toujours plus exigeantes, notamment celle de transformer ses pratiques de façon souple, efficace et économique afin de permettre la formation initiale aussi bien que le perfectionnement en réponse aux demandes que lui adressent un marché du travail soumis à des changements rapides aussi bien que les diverses instances ayant pour mission le développement social.

Mais c'est aussi constater l'effervescence qui marque depuis quelques années l'intérêt de la francophonie à l'égard des NTIC ainsi que l'envergure des initiatives ayant pour but l'appropriation éducative de ces technologies au sein des communautés francophones. De façon maintenant presque banale, les journaux proposent analyses et conseils sur les NTIC, devenues au Québec l'objet de chroniques quotidiennes, tandis que les revues destinées au grand public offrent divers logiciels éducatifs, voire, sous forme d'un livre calqué sur les publications enfantines, une initiation à Internet. Des associations naissent, du «Préau», un centre de ressources et de veille visant à promouvoir l'utilisation des nouvelles technologies éducatives, $\mathrm{au}$ «Net des cartables» et aux CEMIS québécois, sans oublier les réseaux de créateurs de gratuiciels et partagiciels, dont les sites Internet proposent de nombreuses ressources et forums. Mais les manifestations les plus remarquables de cette effervescence sont sans doute les ambitieux programmes mis en œuvre par les grandes organisations de la francophonie, qu'il s'agisse de l'Agence de la francophonie, avec le Consortium international francophone de formation à distance et le Centre international pour le développement de l'inforoute en français, ou de l'Université virtuelle francophone mise sur pied par l'Agence universitaire de la francophonie sous l'égide de l'AUPELF-UREF. L'envergure internationale des projets d'utilisation éducative des NTIC y est manifeste: programmes de formation à distance d'agents d'alphabétisation et de professeurs du secondaire en Afrique subsaharienne, formation au journalisme couplant le vidéo au fax dans les pays francophones, site Internet de «Omar Le Chéri» destiné aux étudiants en journalisme d'Égypte, formation à distance de directeurs d'établissement au Mali; mais aussi de nombreux projets misant sur la collaboration d'universités francophones dans des domaines aussi divers que l'agronomie, le droit, l'économie, les mathématiques, la santé ou les sciences de l'ingénieur.

Pourtant, malgré ces avancées remarquables, de nombreux problèmes se posent encore lorsqu'il s'agit de réaliser des projets visant, grâce aux NTIC, à transformer de façon radicale les pratiques d'établissements d'enseignement, même en formation à distance où le terrain semble pourtant le plus propice à de telles applications. Pour tenter de cerner les difficultés qui s'y présentent, nous nous appuierons sur les travaux de deux collectifs de recherche franco-québécois qui, depuis quelques années, portent leur attention sur le phénomène de l'industrialisation de la formation. 


\section{L'industrialisation de la formation: une perspective critique}

En quoi les règles, pratiques et formes organisationnelles propres à la formation s'apparentent-elles à celles qui structurent le «monde industriel» et sont-elles modelées par ces dernières, du moins de façon tendancielle? Cette interrogation, issue de l'étude des industries culturelles et des services industrialisés, structure l'analyse de l'industrialisation de la formation. À la source, une conviction profonde: les transformations des pratiques éducatives dont les NTIC sont censées être le vecteur témoignent d'une crise de l'appareil scolaire, et cette crise est elle-même inséparable d'une crise économique dans laquelle elle trouve son explication première ${ }^{1}$.

Risquons une schématisation sans doute un peu grossière. Le développement des sociétés industrialisées est souvent rythmé par différentes phases de croissance, chacune marquée par la succession de deux périodes distinctes, soit une période longue de forte croissance, suivie d'une seconde période longue durant laquelle la croissance continue, mais au ralenti. Dans l'histoire contemporaine, l'une de ces phases longues démarre avec la Seconde Guerre mondiale pour s'achever avec le choc pétrolier de 1975-1976: elle est caractérisée par une intervention de plus en plus systématique de l'État, notamment dans les secteurs de l'éducation et de la santé, ainsi que par une tertiarisation explosive de l'emploi et une augmentation spectaculaire des coûts. C'est dans ce contexte que l'on commence à évoquer le projet d'informatiser la société, un projet qui s'affirmera dans toute sa force au début des années quatre-vingt-dix avec la mise en place des autoroutes de l'information. On attend d'elles, en effet, un élargissement des possibilités de formation et une souplesse accrue permettant d'envisager des modalités de formation beaucoup plus flexibles, à la fois dans le cadre des institutions scolaires et en dehors de celles-ci.

Des réorganisations majeures de l'école ont accompagné ces transformations de notre société. Mais si le thème de la crise de l'école fait partie des classiques du discours sur l'éducation, tant sont élevées les attentes que l'on entretient à son endroit, si élevées en fait que leur réalisation est proprement impossible, la crise actuelle dépasse le thème de l'incapacité de l'école à remplir ses responsabilités sociales et économiques pour remettre en cause la viabilité même de l'appareil de formation (Moeglin, 1998c).

Les indices en sont nombreux: malaise des enseignants, absentéisme et abandon des élèves, taux d'échec élevés, incapacité à répondre aux demandes du marché du travail, etc. Ainsi s'est développée dès les années soixante la thèse de la contreproductivité des structures éducatives dont découlerait la contre-productivité économique, ainsi que la fascination pour le mode de fonctionnement de la formation ouverte et permanente, donnée comme exemple de souplesse et d'adaptation aux besoins des individus. Dès lors, un diagnostic s'est imposé dans certains milieux politiques et administratifs, que Tremblay (1998, p. 41) caractérise ainsi:

1. Pour une présentation détaillée de ce point, voir Lacroix (1998). 
Notre système d'éducation doit se transformer pour faire face à la situation. Le taux de décrochage scolaire est trop élevé. Nos méthodes sont inadaptées et inefficaces. Il faut donc tout revoir pour rendre le système performant, moins coûteux et mieux adapté aux nouvelles exigences économiques et sociales. Fort heureusement, les progrès réalisés par les technologies de la communication et de l'information permettent d'entrevoir des solutions novatrices à ces problèmes. L'utilisation adéquate de ces nouvelles technologies va rendre le système d'éducation plus efficace et plus performant, pour peu qu'on veuille s'en donner la peine et s'adapter aux changements nécessaires.

Pour autant, la soumission des institutions d'enseignement aux lois du marché n'est-elle pas tenue pour acquise, comme en témoigne la distinction entre la «University Company» et la "University Society» proposée par Hidalgo (tableau 1). Il n'en reste pas moins que des choix difficiles se posent aux institutions d'enseignement des divers pays:

Les conférences régionales préparatoires ont bien montré que les différents types d'environnement avec lesquels interagissent les institutions d'enseignement supérieur sont en pleine mutation: mondialisation, internationalisation, régionalisation, démocratisation, massification, délocalisation, marginalisation, fragmentation, technologisation. Certains n'hésitent pas à parler de 'crise', au sens étymologique du terme (en grec 'crisis' signifie 'décision') et dans un double sens: de par les changements de structures et donc de visions qui s'y cachent, nous vivons une période 'décisive', c'est-àdire qui conduit à des mutations profondes et sans retour; mais nous vivons aussi et en conséquence une période où des 'décisions' sont nécessaires (Organisation des Nations Unies pour l'éducation, la science et la culture, 1998). 


\section{Tableau 1. Comparaison de deux conceptions pour gérer l'enseignement supérieur (adapté de Hidalgo, 1996)}

\begin{tabular}{|c|c|c|}
\hline Concepts clés & $1^{\text {re }}$ approche: "University Company» & $2^{\mathrm{e}}$ approche: "University Society» \\
\hline $\begin{array}{l}\text { Évaluation de } \\
\text { la qualité }\end{array}$ & $\begin{array}{l}\text { La qualité est déterminée par la capacité du diplômé } \\
\text { de s'insérer lui-même dans le marché du travail. } \\
\text { L'idéologie du marché rentre dans l'institution } \\
\text { d'enseignement supérieur. }\end{array}$ & $\begin{array}{l}\text { La qualité a une signification plus large. Elle est } \\
\text { évaluée à partir de la notion de profitabilité sociale. }\end{array}$ \\
\hline Autonomie & $\begin{array}{l}\text { L'université perd ce qui fait le fondement de son } \\
\text { autonomie, car son activité est déterminée par la } \\
\text { demande induite par le marché. Elle perd donc sa } \\
\text { capacité à décider du type de recherche et d'en- } \\
\text { seignement à mener. }\end{array}$ & $\begin{array}{l}\text { La pensée autonome, c'est-à-dire la recherche de la } \\
\text { vérité et de la rigueur scientifique, est au cœur de } \\
\text { cette approche. Elle ne se laisse donc pas subordon- } \\
\text { ner par l'État ou par des liens particuliers avec des } \\
\text { idéologies ou des partis. }\end{array}$ \\
\hline Accès & $\begin{array}{l}\text { L'accès est conditionné par l'équilibre entre les } \\
\text { demandes des professionnels et les flux d'entrée et } \\
\text { de sortie de l'université. On peut donc s'attendre à } \\
\text { des restrictions dans l'admission; le droit à } \\
\text { l'éducation devient un privilège. }\end{array}$ & $\begin{array}{l}\text { L'éducation est considérée comme un droit } \\
\text { fondamental de tout citoyen. L'université a pour } \\
\text { fonction de former des ressources pour répondre aux } \\
\text { besoins économiques, sociaux, politiques et culturels. } \\
\text { Il s'agit de concilier quantité et qualité, en se basant } \\
\text { sur le principe du mérite. }\end{array}$ \\
\hline Recherche & $\begin{array}{l}\text { La recherche est une mission secondaire. Elle se } \\
\text { justifie seulement si elle peut répondre rapidement } \\
\text { à une demande et à des besoins technologiques. } \\
\text { Les dépenses de recherche et de développement } \\
\text { doivent autant que possible être laissées à des pays } \\
\text { plus développés. }\end{array}$ & $\begin{array}{l}\text { La recherche fondamentale et appliquée et, plus } \\
\text { largement, les connaissances technologiques sont } \\
\text { fondamentales pour deux raisons essentielles: elles } \\
\text { nourrissent l'éducation et la formation; elles rendent } \\
\text { possible un développement durable et autonome. }\end{array}$ \\
\hline $\begin{array}{l}\text { L'éducation } \\
\text { comme priorité }\end{array}$ & $\begin{array}{l}\text { Il s'agit d'une question de priorité. En cas de crise } \\
\text { de ressources, l'enseignement supérieur est moins } \\
\text { prioritaire que les autres niveaux d'éducation. }\end{array}$ & $\begin{array}{l}\text { L'éducation est un processus social de nature } \\
\text { cumulative, une «chaîne éducative ». Le coût } \\
\text { d'opportunité de l'éducation n'est pas une question } \\
\text { interne mais une question liée aux autres secteurs } \\
\text { du développement de la société. }\end{array}$ \\
\hline $\begin{array}{l}\text { L'éducation } \\
\text { comme coût et } \\
\text { investissement }\end{array}$ & $\begin{array}{l}\text { L'éducation est un coût à minimiser, subordonné à } \\
\text { I'équilibre des finances publiques. }\end{array}$ & $\begin{array}{l}\text { L'éducation est un investissement qui permet une } \\
\text { plus grande productivité économique et un mieux- } \\
\text { être culturel et social. }\end{array}$ \\
\hline Efficience & $\begin{array}{l}\text { L'efficience a pour but de rationaliser et de minimiser } \\
\text { les coûts, en fonction d'analyses en termes de coûts- } \\
\text { bénéfices et de calculs économiques. }\end{array}$ & $\begin{array}{l}\text { La notion d'efficience ne peut se résumer à des } \\
\text { critères économiques et comptables, mais à des } \\
\text { critères mixtes de qualité-pertinence-équité, tenant } \\
\text { compte aussi du plus long terme. }\end{array}$ \\
\hline $\begin{array}{l}\text { La formation } \\
\text { comme une } \\
\text { base taxable }\end{array}$ & $\begin{array}{l}\text { L'étudiant reçoit à travers sa formation un «revenu } \\
\text { éducationnel personnel » qui lui rapportera plus tard. } \\
\text { Ce revenu doit donc être taxable. }\end{array}$ & $\begin{array}{l}\text { Le «revenu éducationnel » n'est pas individuel. } \\
\text { C'est un revenu généré en dehors du système } \\
\text { éducationnel, à savoir dans le marché du travail qui } \\
\text { bénéficie des services de l'enseignement supérieur. } \\
\text { La formation n'est donc pas taxable, sous peine } \\
\text { d'encourager l'ignorance. }\end{array}$ \\
\hline $\begin{array}{l}\text { Nature de la } \\
\text { contribution de } \\
\text { I'État }\end{array}$ & $\begin{array}{l}\text { Le système de financement de l'État est un système } \\
\text { en voie de régression et il est même qualifié de } \\
\text { réactionnaire, car le pauvre (qui paie proportion- } \\
\text { nellement plus de taxes) paie pour le riche. Le droit } \\
\text { d'entrée serait en conséquence un mécanisme moins } \\
\text { inéquitable. }\end{array}$ & $\begin{array}{l}\text { Un système de financement avec une intervention } \\
\text { réduite de l'État et l'intervention de droits d'entrée } \\
\text { est bien plus réactionnaire et régressif. Réduire les } \\
\text { iniquités relève avant tout d'une politique de l'État } \\
\text { au niveau de la fiscalité et d'une répartition des } \\
\text { dépenses publiques. }\end{array}$ \\
\hline Équité sociale & $\begin{array}{l}\text { Le système est plus équitable si l'étudiant finance } \\
\text { lui-même sa formation. C'est donc une question } \\
\text { interne à l'institution d'enseignement supérieur, } \\
\text { qui peut ponctionner une partie des droits d'entrée } \\
\text { des plus riches pour financer la formation des } \\
\text { plus pauvres. }\end{array}$ & $\begin{array}{l}\text { Rendre le système plus équitable commande une } \\
\text { politique volontariste plus globale qui implique la } \\
\text { responsabilité des politiques gouvernementales. }\end{array}$ \\
\hline
\end{tabular}


Des pressions s'exercent donc sur ces institutions pour qu'elles se transforment de façon radicale en s'inspirant des principes et des pratiques de gestion de l'entreprise privée. Les attentes à leur endroit sont cependant nombreuses. Il leur faut répondre aux besoins de clientèles diversifiées et aux demandes du marché du travail. Elles doivent être en mesure de trouver de nouveaux débouchés et de s'associer à des partenaires afin de faire face à la diminution de leurs subventions. Elles doivent aussi optimiser l'utilisation de leurs ressources et viser une diminution de leurs coûts de fonctionnement, que ce soit par la décentralisation des opérations ou par la division et la standardisation des tâches. Elles doivent bien sûr intégrer les NTIC dans leurs pratiques d'enseignement et en faire un objet d'étude privilégié. Mais on attend surtout d'elles qu'elles se perçoivent dorénavant à l'image d'entreprises soumises au jeu de la concurrence et qu'elles acceptent d'être gouvernées par des critères de performance quantitatifs et par une exigence de rentabilité.

Bref, des forces sont à l'œuvre pour modeler le champ des activités éducatives selon des principes issus des organisations industrielles, qu'il s'agisse du modèle traditionnel de la grande industrie ou des modèles de production flexible inspirés par la recherche du «juste-à-temps», et que les références soient issues du domaine des industries culturelles ou de celui de l'industrie des services. Rationalisation de l'organisation des activités éducatives, technologisation souvent accompagnée par des investissements importants soumis à des exigences de rentabilisation, recherche de marchés pour les "produits-services ${ }^{2} »$ éducatifs et, plus fondamentalement, intériorisation des principes industriels: tous ces facteurs, qui découlent à la fois de l'influence de politiques publiques visant l'informatisation sociale et d'initiatives des acteurs éducatifs eux-mêmes, concourent à l'industrialisation de la formation avec des intensités et des formes diverses selon les domaines éducatifs mis en cause, de la formation initiale à la formation en entreprise, de la formation en présence aux diverses variantes de la formation à distance et de la formation continue aux activités ludo-éducatives visant le grand public. Il est dès lors facile d'imaginer les bouleversements qu'ils induisent sur les activités éducatives, jusqu'alors caractérisées par le modèle de l'artisanat, et les multiples problèmes qu'ils posent. À défaut d'en faire un examen exhaustif, évidemment impossible dans l'espace limité de cet article, nous nous attacherons à ceux qui trouvent une résonance dans la pratique de la formation à distance.

\section{Des transformations décisives?}

L'intérêt des études sur l'industrialisation de la formation est de mettre en évidence les formes que prend l'introduction de procédés industriels dans les activités éducatives aussi bien que les particularités du secteur éducatif en comparaison du «monde industriel» et les réactions que suscitent ces initiatives. Soulignant la prééminence du rôle de l'État et des politiques publiques, la prévalence de modes de

2. Nous reprenons ici l'heureuse expression de Combès (1998). 
fonctionnement planifiés, la présence de marchés dominés par des prescripteurs et la difficulté de développement de produits éducatifs en grande série - la nature des activités se prêtant plus à l'élaboration de prototypes -, ces études permettent de cerner les difficultés que rencontrent les approches industrielles, mais aussi de mettre en évidence leurs avancées les plus spectaculaires.

Les problèmes qui naissent de la référence industrielle sont bien réels. Par exemple, rien n'est sans doute plus équivoque que les gains de productivité que les NTIC seraient censées permettre. En effet, il est difficile de substituer à une relation d'enseignement en présence une relation médiatisée dans une proportion significative par des machines, étant donné les exigences d'interaction qui accompagnent ces situations d'enseignement. De plus, le temps requis pour l'apprentissage et la médiatisation des enseignements grève singulièrement les gains que l'on pourrait escompter des capacités accrues de stockage et de transmission des NTIC. Autrement dit:

Le temps nécessaire à la préparation des cours, à l'acquisition des connaissances ou à la réalisation des travaux fait souvent pencher la balance en faveur des méthodes traditionnelles, malgré la séduction qu'exercent les possibilités offertes par les nouvelles machines (Tremblay, 1998, p. 53).

On objectera avec raison que la formation à distance permet précisément une telle médiation, que ce soit dans le cadre d'une «classe à distance ${ }^{3}$ » dispensée par vidéoconférence ou dans celui d'un «campus virtuel» offrant un encadrement télématique individuel et des forums de discussion, et on fera justement observer que ces formes d'enseignement médiatisé connaissent un développement important avec l'apport des NTIC. Il n'en reste pas moins cependant, du moins dans le cas de l'enseignement supérieur, que leur utilisation demeure encore assez limitée, soit parce que la classe à distance est perçue comme une solution de dernier recours, lorsqu'il s'avère impossible d'organiser des groupes en présence ${ }^{4}$, soit parce que la médiatisation par le biais du campus virtuel apparaît singulièrement onéreuse en énergies en comparaison d'un mode d'enseignement par correspondance complété par le tutorat téléphonique et éventuellement enrichi par des documents vidéo ou informatiques. C'est ainsi que la réalisation, à la Télé-université, d'un campus virtuel misant sur l'apprentissage collaboratif et mettant en avant des modèles d'enseignement multimédia supportés par de nombreux outils informatiques s'est avérée plus ardue à réaliser qu'on ne l'avait imaginé et que ce campus virtuel n'a pu être développé au rythme prévu, malgré l'ampleur des moyens déployés. Dans les deux cas, l'apprentissage d'une structure d'enseignement complexe et plus exigeante que la structure familière semble avoir joué contre le recours aux nouvelles technologies. On retrouve donc ici une problématique analogue à celle évoquée plus haut, qui

3. Ainsi, la «téléclasse » à laquelle recourent plusieurs constituantes régionales de l'Université du Québec pour desservir leurs bassins de clientèle les plus dispersés se caractérise par une structure de communication pouvant regrouper jusqu'à huit points, grâce à un système combinant une caméra et des micros automatisés, un tableau électronique permettant l'affichage de graphiques en direct et l'utilisation de documents informatiques.

4. Notons cependant que le recours à la téléclasse a permis d'offrir dans plusieurs régions du Québec des cours qui n'auraient pu être donnés autrement, faute d'une clientèle suffisante. 
souligne l'importance des coûts humains associés au développement des projets en regard des bénéfices attendus; cela, bien sûr, sans oublier l'ampleur des coûts techniques, souvent sous-évalués dans un contexte où les budgets sont mesurés au nombre d'étudiants rejoints. Mais il faut également noter, dans le cas du campus virtuel, la résistance qui se manifeste à l'endroit de modes de travail «industriels», lesquels apparaissent étrangers aux pratiques de travail habituelles ${ }^{5}$ et inutilement exigeants étant donné le nombre relativement modeste d'étudiants auxquels s'adressent les cours $^{6}$.

On comprend bien dès lors que, si des difficultés sensibles se présentent dans un domaine que l'on imagine volontiers comme le terrain par excellence de l'utilisation des NTIC - et qui n'hésite pas, le cas échéant, à se présenter comme tel afin d'attirer les subventions publiques -, l'industrialisation de la formation, censée «amorcer la pompe» de la demande solvable, contribuant ainsi à la réussite de la société de l'information, selon l'expression imagée de Miège (1998), est loin d'être aussi assurée qu'on le laisse entendre. Et cela d'autant que le développement le plus visible de l'industrialisation de la formation se situe pour l'instant dans le domaine des produits éducatifs multimédias, faute d'une demande scolaire suffisante. En effet:

Il ne fait pas de doute que les industriels de la culture et de la communication, éditeurs et concepteurs multimédias notamment provisoirement privés d'une vision claire des clients auxquels destiner les produits actuels de la convergence de l'informatique, de l'audiovisuel et des communications, ont de bonnes raisons de se tourner vers les marchés grand public du loisir culturel et de l'autodidaxie. En attendant en effet que se dessine ou que se confirme la possibilité d'applications plus novatrices, ils inscrivent à leurs catalogues les valeurs sûres du patrimoine. En témoigne depuis quelques années la multiplication des éditions de cédéroms et des ouvertures de services en ligne consacrés aux musées, visites, expositions, visites de monuments et de villes, vies et œuvres des grands hommes, etc. (Moeglin, 1998b, p. 115-116).

Pour l'heure donc, l'industrialisation de la formation demeure fort limitée et elle se résume à une augmentation du taux d'équipement ainsi qu'à un recours croissant à des supports médiatisés, lequel est observé particulièrement à l'enseignement primaire et au secondaire. Mais rien n'est encore joué, et tout au plus peut-on avancer, avec Miège (1998), l'hypothèse du développement de quelques marchés de masse coexistant avec une multitude de marchés segmentés proposant des produits

5. Cette résistance mérite d'autant plus d'être remarquée qu'elle intervient dans un milieu où les professeurs tiennent pour acquises la séparation entre la conception des cours et l'exécution des tâches pédagogiques ainsi que la codification des activités pédagogiques, lesquelles apparaissent pourtant à Moeglin (1998a, p. 33) et à certains professeurs «campus » comme antinomiques avec la tradition pédagogique artisanale de l'enseignement et le nécessaire contact entre le professeur et l'étudiant.

6. À cet égard, aucun établissement francophone canadien ne se situe dans la catégorie des "méga-universités» étudiées par John Daniel (1996), dont les cours s'adressent à de vastes clientèles, lesquelles leur permettent d’amortir plus facilement leurs investissements technologiques. 
"ouverts», élaborés sur la base d'une coopération active des enseignants. Aussi rassurante que soit cette perspective, qui suggère l'émergence de nouvelles formes industrielles et l'appropriation des NTIC par les enseignants, elle n'en pose pas moins de nouveaux problèmes, puisqu'elle met en présence deux logiques fort différentes, la première marquée par une recherche de la rentabilité et des économies d'échelle, la seconde par l'offre d'un service accessible au plus grand nombre et visant le développement de la personne.

Il n'y a sans doute pas d'opposition irréductible entre la logique marchande et la logique civique, ce dont témoigne la formation à distance, puisque sa structure de coûts, différente de celle de l'enseignement en présence, l'oblige à amortir les coûts indirects liés à la conception et à la médiatisation des cours sur un nombre minimum d'étudiants, lequel définit leur seuil de rentabilité. En deçà de ce seuil, son coût est donc plus élevé que la formation en présence, tandis que des économies d'échelle deviennent possibles au-delà. Il est donc tout à fait possible, grâce à la formation à distance, d'offrir des cours et des programmes de haute qualité à des coûts moindres que l'enseignement en présence lorsque l'ampleur de la clientèle le permet, et seulement à cette condition. Mais plus élevés sont les coûts de médiatisation et plus lourds sont les coûts de conception associés à l'intégration des technologies dans les cours, plus les exigences de rentabilité risquent de devenir conflictuelles avec les exigences de qualité de la formation lorsque les marchés ciblés sont restreints.

Des pressions s'exercent de ce fait sur les pédagogues pour qu'ils ajustent leurs attentes aux contraintes économiques et, dans certains cas, aux intérêts commerciaux de leurs partenaires. Le projet de création des Presses universitaires multimédias, élaboré par la Conférence des recteurs et des principaux du Québec (CREPUQ, 1996) et sévèrement condamné par la Fédération des syndicats des professeurs des universités concernées (CREPUQ, 1998), en est un exemple révélateur. Ce choc des cultures est sans doute l'un des aspects les plus intéressants que permettent d'observer les études portant sur l'industrialisation de la formation.

\section{Des représentations pédagogiques contrastées}

À défaut, pour les nouveaux médias, de pénétrer en force les pratiques scolaires, et étant donné la résistance du grand public envers ce qui lui rappelle le contexte scolaire dans ses pratiques culturelles ordinaires, on constate en effet que l'industrie des nouveaux médias, par le biais de diverses initiatives, tente d'accréditer la valeur éducative de l'utilisation des médias à domicile, proposant d'y voir l'émergence d'une nouvelle forme d'éducation qui se situerait à la fois dans l'école et en dehors de l'école. On assisterait donc, grâce aux NTIC, à l'avènement de la «société éducative», dans laquelle les formes d'apprentissage feraient appel aux produits offerts par les industries culturelles dans un cadre établi par les structures de formation, mais de façon souple, modularisée et adaptée aux besoins des individus. À mi-chemin entre l'autodidaxie, où l'individu est laissé à lui-même, et l'autoformation, où un cadre 
d'apprentissage le guide, ce modèle du «libre-service» deviendrait un nouvel idéal éducatif .

On remarque qu'un double effort de rationalisation gouverne le recours aux NTIC dans cette perspective. En amont, du côté des institutions, la rationalisation a pour objet la recherche et l'organisation des connaissances afin de permettre l'automatisation des activités répétitives et l'individualisation des parcours d'apprentissage; en aval, du côté des usagers, elle porte plutôt sur l'agencement des ressources éducatives de telle manière qu'ils puissent en disposer de façon optimale, cet agencement pouvant être confié à des agents n'appartenant pas au monde de l'éducation. Mais à ce double effort de rationalisation correspondent aussi deux points de vue différents sur l'apprentissage puisque, dans le premier cas, tous les efforts visent à développer la capacité d'autonomie de l'étudiant en lui faisant acquérir progressivement les habiletés nécessaires, alors que dans le second l'autonomie de l'étudiant est tenue pour acquise et que l'on considère plutôt que celui-ci est le seul responsable de sa démarche d'apprentissage, c'est-à-dire de ses échecs aussi bien que de ses réussites. Ces deux points de vue concurrents sont évidemment assez difficilement conciliables.

En fait, aucun des nouveaux environnements d'apprentissage mis sur pied avec l'apport des NTIC ne pousse cette seconde rationalisation à l'extrême puisqu'une certaine assistance est presque toujours rendue disponible. Toutefois, les premières observations confirment qu'à côté de lieux de formation où la coprésence formateur/apprenant est maximale et de lieux basés sur l'autoformation il existe maintenant des lieux de type «libre-service» (essentiellement des centres de ressources) où les étudiants bénéficient d'une aide minimale et se retrouvent dans une situation de relative solitude devant une offre de produits surabondante (Fichez, 1998). Le risque, dans cette perspective, est donc qu'au nom d'une philosophie de l'«apprenant-roi» héritée du secteur marchand se développent des structures d'apprentissage où la médiation humaine serait remplacée par des médiations techniques à l'image de celles que développent les institutions de formation à distance, dans lesquelles l'apprenant serait largement laissé à lui-même. Or, à défaut de savoir ce qu'il doit apprendre et de pouvoir reconnaître ses compétences, à défaut de pouvoir repérer quelles ressources lui seront utiles, et selon quel agencement. Faute d'un support adéquat pour ce faire, l'apprenant risque fort de perdre cette motivation que les systèmes multimédias sont censés créer et entretenir chez lui. Les mêmes difficultés se posent quant à l'organisation des cursus de formation, et l'on peut difficilement imaginer qu'un étudiant seul compose son «menu» en juxtaposant librement des éléments de formation suivis dans différents établissements d'enseignement selon ses besoins et ses modalités d'apprentissage.

Ces craintes sont certes légitimes, notamment dans la mesure où certains orga nismes ministériels proposent une telle déréglementation et tendent vers l'instauration d'un libre-service éducatif ${ }^{8}$. Elles méritent cependant quelques commentaires.

7. Pour une présentation détaillée de la notion de «libre-service», voir Moeglin (1998b).

8. C'est le cas du rapport Quéré (1994), qui propose un enseignement universitaire sur mesure et envisage la disparition des enseignants avec le développement des environnements médiatisés. 
Ainsi la préoccupation envers les conditions de l'autonomie des étudiants touche-telle une corde très sensible dans le contexte de la formation à distance où l'on constate des taux d'abandon parfois plus élevés que dans l'enseignement en présence, lesquels constituent une source de préoccupation récurrente, aussi bien pédagogique qu'économique. Car si les étudiants qui réussissent témoignent d'un degré d'autonomie élevé, on se doute bien que cette autonomie constitue, à rebours, le talon d'Achille de la formation à distance, surtout lorsque l'étudiant travaille seul à domicile $^{9}$ et que les exigences des cours lui apparaissent plus élevées que celles des cours suivis sur campus ${ }^{10}$. À cet égard, la disponibilité d'une médiation efficace apparaît effectivement comme un facteur critique afin de réduire la concurrence entre le travail, les études, la vie familiale et les loisirs, qui est le lot de la plupart des étudiants adultes ${ }^{11}$, ou du moins d'en tirer parti pour aménager des environnements d'apprentissage plus efficaces. Pour sa part, une institution telle que la Télé-université sait depuis toujours le rôle vital qu'exercent ses ressources de tutorat, souvent considérées par les étudiants comme leurs véritables professeurs, et elle sait bien également qu'une véritable médiation ne peut se résumer à une conférence télématique vouée à la socialisation ${ }^{12}$ mais doit s'inscrire également dans le processus d'enseignement lui-même.

D'autres facteurs que la médiation déterminent d'ailleurs la persévérance de l'étudiant, par exemple la mise à jour des contenus présentés, un point particulièrement sensible quand ces contenus traitent de technologie, mais aussi, de façon plus générale, la pertinence des contenus par rapport aux situations professionnelles et la qualité de l'interrelation entre la théorie et la pratique qui est proposée dans le cadre des cours. À cet égard, et de façon un peu divergente avec le diagnostic que propose l'analyse de l'industrialisation de la formation, il ne nous semble pas assuré que la perspective d'un enseignement sur mesure bâti en s'appuyant sur les situations professionnelles des étudiants soit irréconciliable avec une organisation pédagogique satisfaisante. Comme le démontre l'exemple d'un séminaire ${ }^{13}$ développé dans le cadre de l'École de technologie de l'information, il est en effet possible de structurer sur une base individualisée une analyse de contextes professionnels variés tout en permettant des mises en commun autour de thématiques d'intérêt général, grâce à une démarche rigoureuse faisant appel à une utilisation intensive des NTIC (voir le

9. Les pratiques de médiation en formation à distance varient ici de façon très marquée, allant du contact téléphonique ou télématique (Télé-université) à la coprésence visuelle (téléclasse), en passant par des combinaisons de ces moyens (TéléÉducation du Nouveau-Brunswick).

10. C'est l'une des constatations récentes de la Télé-université, dont une bonne partie de la clientèle vient maintenant des autres universités, leurs étudiants venant chercher à distance les quelques crédits libres auxquels ils sont autorisés dans le cadre de leurs programmes.

11. Ce dont témoignent sans doute les efforts de I'Université du Québec à Montréal, qui, parallèlement à la mise en place de nombreux services high-tech, a développé des services de soutien à l'apprentissage tels que le monitorat par des étudiants avancés, le mentorat, le parrainage et les réseaux de pairs, réduisant ainsi de facon sensible le taux d'abandon de ses étudiants.

12. La mise en relation télématique des étudiants s'avère cependant porteuse de potentialités pédagogiques, telle que l'émergence de réseaux de collaboration permettant un «apprentissage coopératif » dont la systématisation apparaît prometteuse (Hotte, 1998).

13. II s'agit du cours ETI 6001 Séminaire sur les applications des technologies de l'information, qui propose d'analyser l'impact des technologies de l'information dans différents secteurs de la société (ressources naturelles, industrie manufacturière, transport et communication, commerce, santé et services sociaux, culture, divertissement, éducation et formation) et de développer une réflexion critique à cet égard. 
tableau 2 et les figures A et B). Ce modèle de séminaire médiatisé porte à croire que la formation à distance n'est pas nécessairement un cheval de Troie de l'invasion technologique et de la logique marchande, mais peut au contraire s'avérer un laboratoire fécond pour l'apprivoisement pédagogique bien tempéré des technologies dans le contexte d'apprentissages ouverts. On comprend cependant que la conception d'un tel environnement d'apprentissage est singulièrement exigeante. De la même façon, l'entropie qui résulterait de la juxtaposition aléatoire de contenus d'apprentissage dans des cursus individualisés ne doit pas être tenue pour certaine, dans la mesure où des organismes veillent à la qualité de structuration pédagogique des programmes et interviennent en ce sens, comme l'a déjà fait le Conseil des universités du Québec (1986) et comme le font les différents organismes de contrôle de la qualité et de la pertinence des programmes universitaires dont sont dotées plusieurs provinces canadiennes.

Tableau 2. Structuration du séminaire ETI 6001

\begin{tabular}{|c|c|c|c|c|c|}
\hline Tâches & Résultats attendus & $\begin{array}{l}\text { Modalités } \\
\text { de travail }\end{array}$ & Semaine & Échanges & Notation \\
\hline \multicolumn{6}{|c|}{$\begin{array}{c}\text { Activité } 1 \text { - Démarrage du séminaire } \\
\text { Apprivoiser l'environnement virtuel du séminaire et planifier le travail }\end{array}$} \\
\hline $\begin{array}{l}\text { 1. Découverte de } \\
\text { l'environnement }\end{array}$ & $\begin{array}{l}\text { Appropriation des ressources } \\
\text { Rencontre des participants }\end{array}$ & Individuel & 1 & $\begin{array}{l}\text { Forum Dépanne } \\
\text { (au besoin) } \\
\text { Forum Coordination }\end{array}$ & Aucune \\
\hline $\begin{array}{l}\text { 1. Préparation au } \\
\text { séminaire }\end{array}$ & Planification du travail & Individuel & 1 & Forum Coordination & Aucune \\
\hline \multicolumn{6}{|c|}{$\begin{array}{c}\text { Activité } 2 \text { - État des lieux } \\
\text { Appréhender la complexité des innovations et de leurs applications dans le domaine des technologies de l'information }\end{array}$} \\
\hline $\begin{array}{l}\text { 1. Recensement } \\
\text { des innovations }\end{array}$ & $\begin{array}{l}\text { Autoformation à NOMINO } \\
\text { Notes de lecture et signets }\end{array}$ & Individuel & 1,2 & Forum Nomino & Aucune \\
\hline $\begin{array}{l}\text { 1. Synthèse } \\
\text { personnelle }\end{array}$ & $\begin{array}{l}20 \text { fiches d'information } \\
\text { Rapport synthèse «État des } \\
\text { lieux» }\end{array}$ & Individuel & 3,4 & $\begin{array}{l}\text { Forum Nomino } \\
\text { Forum Coordination } \\
\text { Vitrine }\end{array}$ & $15 \%$ \\
\hline $\begin{array}{l}\text { 1. Analyse de } \\
\text { groupe }\end{array}$ & Discussion asynchrone & En groupe & $4,5,6,7$ & Forum $T I C$ & $5 \%$ \\
\hline \multicolumn{6}{|c|}{$\begin{array}{c}\text { Activité } 3 \text { - Analyse sectorielle } \\
\text { Analyser un secteur spécifique d'application des technologies de l'information selon un point de vue technologique, économique, } \\
\text { éthique, social et culturel }\end{array}$} \\
\hline $\begin{array}{l}\text { 1. Élaboration } \\
\text { d'une stratégie } \\
\text { de recherche }\end{array}$ & $\begin{array}{l}\text { Organisation du travail } \\
\text { d'équipe }\end{array}$ & En équipe & 5 & Courriel et ICQ & Aucune \\
\hline $\begin{array}{l}\text { 1. Analyse } \\
\text { d'un secteur } \\
\text { d'application }\end{array}$ & Rapport d'analyse sectorielle & En équipe & $\begin{array}{l}5,6,7 \\
8,9\end{array}$ & Courriel et ICQ & $20 \%$ \\
\hline $\begin{array}{l}\text { 1. Présentation de } \\
\text { groupe }\end{array}$ & $\begin{array}{l}\text { Présentation PowerPoint des } \\
\text { équipes } \\
\text { Table ronde }\end{array}$ & En groupe & 9,10 & $\begin{array}{l}\text { Vitrine } \\
\text { Conférence téléphonique }\end{array}$ & $10 \%$ \\
\hline
\end{tabular}


Tableau 2. Structuration du séminaire ETI 6001 (suite)

\begin{tabular}{|c|c|c|c|c|c|}
\hline Tâches & Résultats attendus & $\begin{array}{l}\text { Modalités } \\
\text { de travail }\end{array}$ & Semaine & Échanges & Notation \\
\hline \multicolumn{6}{|c|}{$\begin{array}{c}\text { Activité } 4 \text { - Étude de cas } \\
\text { Intégrer les notions conceptuelles relatives au domaine des technologies de l'information à la pratique concrète sur le terrain, } \\
\text { par l'étude d'un cas particulier }\end{array}$} \\
\hline $\begin{array}{l}\text { 1. Planification du } \\
\text { projet }\end{array}$ & $\begin{array}{l}\text { Plan de réalisation } \\
\text { Approche méthodologique } \\
\text { Proposition }\end{array}$ & Individuel & $\begin{array}{l}4,5,6 \\
7,8\end{array}$ & Forum Coordination & Aucune \\
\hline $\begin{array}{l}\text { 1. Collecte } \\
\text { d'informations }\end{array}$ & Documentation du cas & Individuel & $\begin{array}{l}9,10,11 \\
12,13\end{array}$ & Forum Coordination & Aucune \\
\hline $\begin{array}{l}\text { 1. Réalisation de } \\
\text { l'étude de cas }\end{array}$ & Rapport de I'étude de cas & Individuel & $\begin{array}{l}13,14 \\
15\end{array}$ & Forum Coordination & Aucune \\
\hline \multicolumn{6}{|c|}{$\begin{array}{c}\text { Activité } 5 \text { - Synthèse du séminaire } \\
\text { Effectuer un retour sur la démarche d'apprentissage et dégager une vision critique de la problématique des technologies } \\
\text { de l'information }\end{array}$} \\
\hline 1. Bilan personnel & $\begin{array}{l}\text { Objectivation de la démarche } \\
\text { d'apprentissage } \\
\text { Réflexion critique sur les TI } \\
\text { Appréciation du séminaire }\end{array}$ & Individuel & 14 & $\begin{array}{l}\text { Forum Coordination } \\
\text { Forum TIC }\end{array}$ & $5 \%$ \\
\hline $\begin{array}{l}\text { 1. Synthèse } \\
\text { collective }\end{array}$ & $\begin{array}{l}\text { Opinions } \\
\text { Discussion asynchrone et } \\
\text { synthèse du cours }\end{array}$ & En groupe & 14,15 & Forum $T I C$ & $5 \%$ \\
\hline
\end{tabular}

Figure A. Séminaire ETI 6001 (Présentation générale)

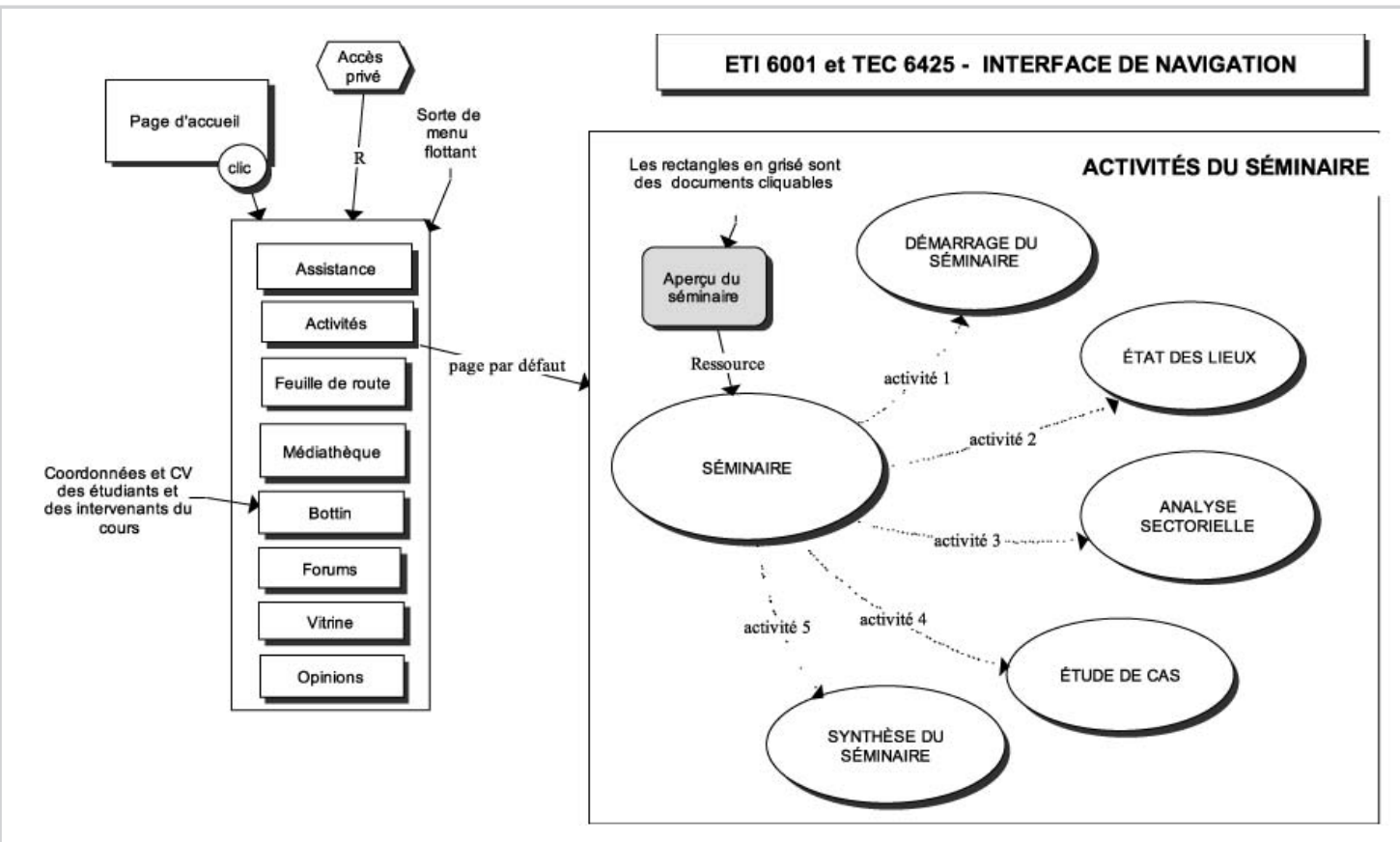


Figure B. Séminaire ETI 6001 : I’activité 2 (État des lieux)

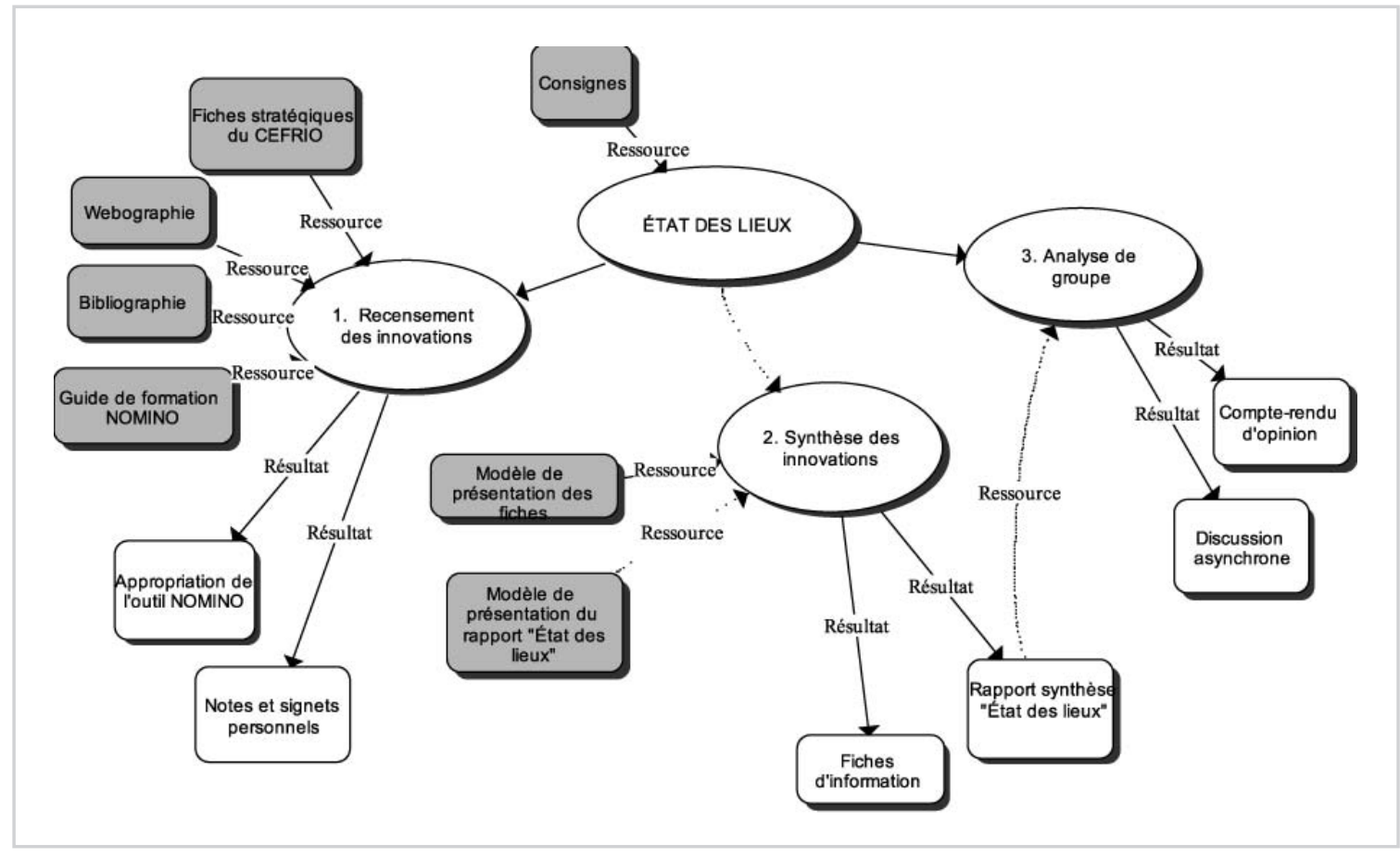

Au total, il s'agit donc de faire en sorte que le recours aux NTIC s'accompagne d'une démarche éducative à la fois ouverte et rigoureuse, permettant la mise en relation des théories et des pratiques, et accompagnée d'une médiation efficace, portant à la fois sur les contenus et les processus d'apprentissage. Ces exigences conditionnent étroitement la motivation et la persistance des étudiants dans leur apprentissage.

\section{Un terrain propice?}

L'enjeu n'est cependant pas seulement pédagogique, ou du moins pas seulement dans la perspective pédagogique qu'abordent les études de l'industrialisation de la formation. Ainsi, il y a lieu de se demander au préalable dans quelle mesure les enseignements peuvent être médiatisés et quel peut être l'avantage marginal du recours aux NTIC. À cet égard, la vitrine que constituent la diffusion internationale de cours d'initiation à Internet ou l'exploration d'univers virtuels ne doit pas faire oublier que certaines disciplines sont encore assez peu touchées ou présentent des difficultés de médiatisation importantes. Ainsi, l'illustration de l'histoire des civilisations ou de l'histoire des arts constitue sans contredit un terrain de prédilection pour les technologies du multimédia. Mais l'apprentissage de l'interprétation musicale, de la philosophie de Kierkegaard ou des techniques d'écriture est une tout autre paire 
de manches. L'apprentissage à distance des techniques chirurgicales est certes maintenant envisageable avec l'apparition des premiers robots chirurgicaux, mais on imagine bien que son développement risque d'être passablement lent. Et bien que l'on ait déjà tenté de simuler la conversation psychothérapeutique ${ }^{14}$, ni la psychanalyse virtuelle, ni l'analyse du test de Rorschach assistée par ordinateur ne sont pour demain. La médiation pédagogique humaine a donc de beaux jours devant elle, avant l'avènement de la machine de Turing ${ }^{15}$.

Bref, la formalisation et l'automatisation des connaissances sont nécessairement limitées, et si les NTIC offrent de puissantes capacités d'archivage, d'organisation et d'interrelation, ces dernières sont d'une piètre utilité lorsque les connaissances sont de l'ordre de la construction sociale ou lorsqu'il s'agit de développer des habiletés motrices ou créatives. Cela permet bien sûr de comprendre les défis auxquels la Téléuniversité a dû faire face dans la mise sur pied de son campus virtuel, auquel était associée une impressionnante ingénierie de formation multimédia. Mais cela permet également de comprendre l'attachement que manifestent beaucoup de professeurs à l'égard de l'enseignement présentiel, dont témoignent de façon très nette les analyses de l'industrialisation de la formation ${ }^{16}$, ainsi que l'intérêt que présentent, à titre de succédané, la vidéoconférence ou la téléclasse. Comme on le voit, les exigences pédagogiques sont nombreuses. Il faut en effet tout à la fois permettre l'apprentissage individuel et les interactions avec le professeur ou entre les étudiants, donner accès aux données disponibles sur Internet, favoriser le travail à domicile au rythme de l'étudiant, mais donner réponse à ses questions aussi rapidement que possible, et ainsi de suite. Bien entendu, la combinaison de différents médias peut s'avérer très utile, de même que la combinaison entre l'interaction synchrone et le travail asynchrone. Mais les coûts de tels environnements s'avèrent vite élevés, aussi bien pour l'établissement d'enseignement que pour l'étudiant, et des compromis doivent être trouvés entre ces diverses exigences. La mise à contribution des NTIC, pour le moment, se trouve ainsi limitée par son objet même.

D'autres facteurs jouent un rôle qui, pour être discret, n'en est pas moins déterminant. Ainsi en est-il de la formation aux technologies, dont nul ne nie la nécessité. Mais il est assez troublant de constater, bien souvent, que l'expérimentation des NTIC dans le cadre de la préparation des activités d'enseignement est elle aussi souvent éludée et que l'on tient en quelque sorte pour acquis que professeurs et enseignants intégreront cette tâche dans le cadre de leurs activités habituelles ou

14. On se souviendra du logiciel Éliza, qui imitait les reformulations de la thérapie rogérienne.

15. Dans son ouvrage Computing Machinery and Intelligence (1950), Alan Turing, imaginait un «jeu de l'imitation » dans lequel un humain et un ordinateur seraient interrogés de telle façon que l'interrogateur ne pourrait dire qui lui répondait, la communication s'effectuant par messages textuels. Turing prétendait que si l'interrogateur ne pouvait discerner à qui il s'adressait, on ne pouvait refuser de considérer l'ordinateur comme intelligent. Ce jeu de l'imitation a été par la suite appelé le test de Turing.

16. La possibilité d'ajustement aux réactions des étudiants, que ce soit durant ou après le cours, est effectivement essentielle. Mais on reconnaîtra que les situations d'enseignement en présence pèchent souvent à cet égard, particulièrement lorsque les groupes sont nombreux, et que l'interaction est parfois très rudimentaire. C'est d'ailleurs cette constatation, ainsi que le caractère répétitif des questions posées, qui a amené le développement de l'enseignement assisté par ordinateur et des FAQ, lesquelles peuvent être d'excellents outils pédagogiques. 
dans leurs moments de loisir ${ }^{17}$. Or, cette mise en application des connaissances acquises lors des séances de formation, qui coïncide souvent avec un rodage des nouveaux systèmes, constitue précisément la partie la plus exigeante de l'apprivoisement des NTIC, surtout lorsque les usagers ne sont pas technophiles, que ce soit par crainte, méfiance ou manque d'intérêt. Il s'agit donc de prévoir explicitement une plage à cet effet dans l'aménagement des temps de travail ainsi qu'un accompagnement approprié, sans oublier l'assistance technique. On voit ici la difficulté de l'entreprise dans le contexte de restrictions budgétaires qui accompagne souvent l'introduction des NTIC, puisqu'il s'agit de consentir à un investissement humain important, outre l'investissement technique, en vue de gains de productivité ou de qualité pédagogique pas toujours faciles à déterminer.

Assurer la crédibilité des changements envisagés aux yeux des prescripteurs pédagogiques qui, à l'observation des expérimentations des innovateurs, estiment les gains possibles pour les institutions, et négocier les politiques internes, notamment avec les syndicats et associations: tels sont les déterminants de l'acceptabilité des NTIC dans les pratiques d'enseignement. On reconnaît là les conditions de l'institutionnalisation du changement, et notamment de cette phase de pré-institutionnalisation que Tolbert et Zucker $(1995,20)$ appellent l'«habitualisation». En effet:

Organizations experiencing a problem may, as part of their search for solutions, also consider solutions developed by others. Imitation may follow, but there is little sense of the necessity of this among organizational decision makers, since there is no consensus on the general utility of innovation. Hence, adoption can be predicted largely by characteristics that make a change technically and economically viable for a given organization and by internal political arrangements that make organizations more or less receptive to change processes.

Le terrain, on le voit, demande donc d'être labouré et engraissé. Et sans doute la difficulté principale que rencontrent les établissements d'enseignement dans leur démarche d'ouverture aux NTIC consiste-t-elle à trouver des compromis satisfaisants entre les performances pédagogiques des systèmes, leurs contraintes techniques et les disponibilités financières dans le cadre des budgets réguliers d'enseignement. À cet égard, le succès que connaissent certains logiciels de création d'environnements d'apprentissage Internet a sans doute valeur d'exemple ${ }^{18}$ et permet de penser que si certaines implantations technologiques s'avèrent laborieuses, voire improbables, d'autres sont probablement en passe d'être institutionnalisées. Bien sûr, la médiatisation est ici partielle, puisque les étudiants continuent à travailler à partir de leurs manuels, n'utilisant Internet que pour consulter des sources d'information complémentaires, échanger avec leur professeur, prendre connaissance

17. Ce manque de disponibilité risque par exemple d'être tout à fait problématique dans le cas d'une enseignante qui a la charge d'activités domestiques à son retour au domicile.

18. Ainsi, I'Université d'Alberta, qui offrait à l'été 1998 plus de 80 cours dans diverses disciplines médiatisés à l'aide du logiciel WebCT, en offre maintenant plus de 150. Ce logiciel est largement utilisé au Canada et aux États-Unis, ainsi que par plusieurs institutions en Australie, à Hong Kong, en Grande-Bretagne et en Belgique. 
des travaux à effectuer et acheminer ces travaux. Et, bien sûr, ces logiciels n'offrentils qu'une gamme assez limitée de fonctionnalités, c'est-à-dire essentiellement un éditeur HTML associé à un générateur d'exercices et à un gestionnaire de conférences électroniques. Mais peut-être est-ce précisément cette parcimonie qui explique leur succès, à la fois en raison de leur facilité d'apprentissage, de leur coût modeste, de leur polyvalence et du fait qu'ils viennent heureusement compléter l'enseignement traditionnel.

Il faut sans doute y voir de plus près et évaluer l'impact de ces technologies de façon plus profonde. Mais une seconde observation s'impose, qui a cette fois trait au mode de travail qu'amène le recours aux NTIC. Souvent, en effet, l'innovation a été associée à des projets de réalisation multimédia de grande envergure reposant sur la contribution de multiples spécialistes. Or, il semble bien au contraire, dans le cas de l'enseignement supérieur, qu'un tel scénario soit doublement handicapé, à la fois en raison des difficultés de coordination associées à ces projets et aussi parce que l'investissement pédagogique n'est pas comptabilisé dans les dossiers de promotion des professeurs comme le sont les publications et les recherches. Le tout s'ajoutant sans doute aux habituelles difficultés de cohabitation de statuts professionnels distincts comme en ont connu les productions audiovisuelles. À l'inverse, le succès des logiciels de médiatisation «modestes» s'explique-t-il aussi par l'autonomie qu'ils laissent aux professeurs, qui peuvent préparer leur cours à partir de leur domicile et avec l'aide d'un assistant, généralement un étudiant expérimenté, comme ils le faisaient auparavant pour la préparation de leurs manuels.

Propice, le terrain de la formation l'est donc peut-être, pour la mise à contribution des NTIC, mais d'une manière elle aussi fort contrastée selon les lieux, même dans un champ comme la formation à distance, et de nombreux facteurs spécifiques y exercent leur influence. Dans ces conditions, l'éventuelle industrialisation de la formation qui peut l'affecter risque d'être assez imprévisible.

\section{Une industrialisation insolite}

Comme on pouvait s'y attendre, l'heure de la production de masse de "produitsservices» éducatifs n'est pas encore venue, du moins pas dans le domaine de la formation à distance. Pourtant, la multiplication d'environnements d'apprentissage médiatisés à l'aide de quelques logiciels qui jouent le rôle de gabarits suggère que l'industrialisation, sous la forme de la standardisation, est bel et bien en marche et qu'elle préfigure peut-être la naissance d'une industrie du "manuel électronique», c'est-à-dire un livre ouvert sur des réseaux d'information et de communication selon un cadre pédagogique prédéterminé. Gageons qu'elle touchera plus probablement les cours de grande diffusion et dans une moindre mesure les cours dont le contenu est plus spécialisé. L'industrialisation risque donc de passer d'abord par les processus de médiatisation pour s'étendre à différents types de contenus, par exemple les cours de première année de baccalauréat ou les cours culturels de grande diffusion. 
L’originalité de ce modèle est d'autant plus grande que dans le cas des logiciels de création d'environnements d'apprentissage sur Internet, le processus d'enseignement médiatisé ne repose pas sur la division du travail comme le suggère le modèle classique de l'industrialisation, hérité de la grande industrie, mais plutôt sur l'autonomie de l'enseignant, souvent aidé d'un assistant ${ }^{19}$. On peut donc se demander si la combinaison gagnante n'est pas, pour l'instant, celle de l'«artisanat industriel», aussi opposées que soient en principe ces deux références. La résolution de ce paradoxe réside probablement dans une observation fine des modèles de production industriels marqués par la recherche du «juste à temps» et de la "qualité totale», où les opérateurs, dont le niveau de formation est souvent élevé, jouissent d'une grande autonomie dans l'exécution de leur travail et dans l'organisation de leurs tâches, avec une chaîne industrielle composée d'ensembles de tâches exécutées de façon relativement flexible.

Cette évolution est sans doute rassurante puisqu'elle montre que malgré les caractéristiques «industrielles» associées à la structure de coûts de la formation à distance, l'arrivée des NTIC ne se traduit pas nécessairement par une atomisation de la chaîne d'enseignement, même quand un investissement public important est associé à un modèle de multimédiatisation fortement inspiré par l'ingénierie. Et il est intéressant de constater, dans le cas d'une institution telle que la Télé-université où la séparation entre le professeur et l'étudiant s'était imposée comme une norme ${ }^{20}$, que le recours aux NTIC est perçu comme un moyen privilégié pour permettre le retour à une forme d'enseignement plus organique ${ }^{21}$.

Il est encore trop tôt pour tirer des conclusions de ces pratiques naissantes. Mais les premiers efforts semblent être caractérisés par la recherche de nouveaux modes d'enseignement laissant une plus large place à l'appropriation des connaissances par l'étudiant, plutôt qu'à leur simple diffusion. Reconnaissons toutefois que ces efforts sont coûteux, qu'il n'est pas facile de mettre sur pied des modèles de pédagogie "appropriative», et que d'ailleurs les diverses disciplines s'y prêtent avec plus ou moins de bonheur. Sans doute certaines formes d'utilisation des nouvelles technologies sont-elles peu novatrices sur le plan pédagogique et sans doute certaines promesses pédagogiques donnent-elles lieu à des réalisations quelque peu caricaturales (Moeglin, 1998c). Mais il est vrai que l'innovation dans les pratiques est rarement simultanée avec l'innovation technique, et sans doute faut-il témoigner à cet égard d'une certaine patience et d'une bonne dose d'optimisme.

L'introduction des NTIC se traduit-elle par une régression pédagogique (Moeglin, 1998c, p. 226-227)? Ce jugement un peu sévère est sans doute prématuré, tant sont diverses les expériences et les réalisations. Et cela même sur un terrain tel

19. Comme le montre le cas du certificat en traduction médiatisé par le Collège universitaire de Saint-Boniface.

20. Cette norme s'est d'ailleurs imposée progressivement, puisqu'à ses origines la Télé-université recourait systématiquement à des ateliers dans le cadre de ses cours.

21. II faut cependant comprendre que cette relation organique n'a de sens que si elle s'accompagne d'un réajustement continu des contenus de cours en fonction des problèmes pédagogiques rencontrés. En ce sens, l'intervention des technologues de l'éducation dans le modèle traditionnel de la Télé-université trouve sa justification dans le souci de réduire à la source les problèmes pédagogiques en se basant sur l'expérience des cours antérieurs. 
que la formation à distance, où cohabitent, d'une part, les «téléservices » qui sont des activités délocalisées réalisées en temps réel et à distance, de manière ponctuelle et discontinue, généralement sur une base collective et, d'autre part, des activités individuelles synchrones et asynchrones comme celles que pratique la Télé-université. De même, il est frappant de constater que de deux formes d'enseignement assez apparentées, celle du programme «Europace» et celle de la «téléclasse», la première a été abandonnée après quelques mois, alors que la seconde ne cesse de se développer depuis quelques années. Il faut donc y voir plus clair et trouver une base de comparaison commune entre toutes ces expériences, c'est-à-dire commencer sans doute par caractériser les attributs permettant d'évaluer la qualité pédagogique des nouveaux environnements médiatisés.

Mais une chose est certaine. Tout comme la formation à distance à ses débuts, les NTIC se voient investies d'un grand nombre d'attentes dont la réalisation est relativement difficile de façon simultanée. Mise à jour rapide des contenus, exploitation des ressources technologiques les plus récentes, individualisation du rythme et des parcours d'apprentissage, voire du style d'apprentissage, interaction avec des pairs et des ressources expertes, apprentissage coopératif, accessibilité accrue, économies d'échelle et réduction des coûts: toutes ces attentes dont le bon sens indique, de toute évidence, la très improbable coexistence ne figurent pas moins dans l'imaginaire des nouvelles technologies pédagogiques, et depuis fort longtemps. On retrouve dans ce paradoxe la traditionnelle ambiguïté des citoyens à l'égard du politique, qui les amène à nourrir de nombreuses attentes, justifiant ainsi les promesses des programmes des différents partis, tout en sachant fort bien qu'elles ne pourront être toutes réalisées parce qu'elles sont mutuellement opposées. Cela explique notamment pourquoi des décisions apparemment contradictoires peuvent découler d'une même lecture des attentes initiales, selon que l'accent est mis sur une attente plutôt que sur une autre (Jones, 1994). La question est donc de savoir, test de réalité ultime, quelles attentes doivent s'imposer aux autres.

Le choix est difficile. Mais en visant l'intérêt premier des étudiants, dans un souci d'ajuster les apprentissages théoriques aux apprentissages pratiques, de combiner des périodes d'apprentissage individuel et d'interaction et de faire de la technologie une consommation modérée, des solutions devraient s'imposer graduellement. Elles seront probablement guidées par des principes de souplesse, de modularité et d'économie, et sauront ainsi composer avec les limites de la réalité et de la créativité. 


\section{Références bibliographiques}

COMBÈS, Y. (1998). Produits-services éducatifs hors l'école. Dans P. Moeglin (dir.), L'industrialisation de la formation - État de la question (p. 151-170). Paris: Centre national de documentation pédagogique.

CONSEIL DES UNIVERSITÉS (1986). La formation courte dans les universités. Québec: Conseil des universités, 69 p.

CREPUQ (1996). Les presses universitaires multimédias. Montréal: Conférence des recteurs et des principaux du Québec, 31 p.

CREPUQ (1998). Actes du colloque "Pour un meilleur accès à l'excellence». Montréal: Conférence des recteurs et des principaux du Québec, 140 p.

DANIEL, J. S. (1996). Mega-universities and Knowledge Media: Technology Strategies for Higher Education, London: Kogan Page, 212 p.

FICHEZ, É. Industrialisation contre médiation. Dans P. Moeglin (dir.), L'industrialisation de la formation - État de la question (p. 133-150). Paris: Centre national de documentation pédagogique.

HIDALGO, J. C. (1996). Financing Higher Education. Havana: CRESALC, UNESCO. Document elaborated for the Regional Conference on Policies and Strategies for the Transformation of Higher Education in Latin America and the Caribbean.

HOTTE, R. (1998). Modélisation d'un système d'aide multiexpert pour l'apprentissage coopératif à distance. Thèse de doctorat, Université Paris 7, juin.

JONES, B. D. (1994). Reconceiving Decision-Making in Democratic Politics Attention, Choice and Public Policy. Chicago : The University of Chicago Press, $277 \mathrm{p}$.

LACROIX, J.-G. (1998). Au cœur des recompositions économiques, la crise de l'école. Dans P. Moeglin (dir.), L'industrialisation de la formation - État de la question (p. 173-195). Paris: Centre national de documentation pédagogique.

MIĖGE, B. (1998). Voies multiples. Dans P. Moeglin (dir.), L’industrialisation de la formation - État de la question (p. 95-106). Paris: Centre national de documentation pédagogique.

MOEGLIN, P. (1998a). Introduction. Dans P. Moeglin (dir.), L'industrialisation de la formation - État de la question (p. 7-36). Paris: Centre national de documentation pédagogique.

MOEGLIN, P. (1998b). Entre service et self-service. Dans P. Moeglin (dir.), L'industrialisation de la formation - État de la question (p. 107-131). Paris: Centre national de documentation pédagogique. 
MOEGLIN, P. (1998c). Industrialisation, crise, réindustrialisation. Dans P. Moeglin (dir.), L'industrialisation de la formation - État de la question (p. 207-247). Paris: Centre national de documentation pédagogique.

ORGANISATION DES NATIONS UNIES POUR L'ÉDUCATION, LA SCIENCE ET LA CULTURE (1998). L'enseignement supérieur au XXIe siècle - Vision et actions. Document de travail ED-98/CONF.202/CLD.23. Paris: Unesco, août, 64 p.

QUÉRÉ, M. (1994). Vers un enseignement sur mesure. Paris: Ministère de l'Enseignement supérieur et de la Recherche, Direction générale des enseignements supérieurs, juin.

TOLBERT, P. S. et ZUCKER, L. G. (1995). Institutional Analyses of Organizations: Legitimate but not Institutionalized. Présentation à l'American Sociological Meeting, Washington, DC, $46 \mathrm{p}$.

TREMBLAY, G. (1998). Une approche pertinente? Dans P. Moeglin (dir.), L'industrialisation de la formation - État de la question (p. 39-59). Paris: Centre national de documentation pédagogique. 